\title{
Editorial
}

\section{Modelos de Decisão aplicados à Saúde: teoria e prática}

\author{
Ronei Marcos de Moraes ${ }^{1}$ \\ Rackynelly Alves Sarmento Soares ${ }^{2}$
}

O processo de se escolher uma ação dentre várias possíveis visando a solução, mitigação ou prevenção de problemas é denominado de tomada de decisão. Esse processo é desencadeado sempre que existe um problema a ser resolvido, seja devido ao estado atual das coisas ser diferente do estado desejado, seja pela quebra de paradigmas estabelecidos, ou ainda pela busca por uma maior eficiência ou pela necessidade de redução de custos, entre outros.

De fato, a própria evolução natural do ser humano o tornou um "tomador de decisões", sendo que diariamente tomamos dezenas de decisões complexas e baseadas em várias informações diferentes, mas sempre com base na subjetividade e exepriências individuais. Por muitos séculos, essa foi a base do processo de tomada de decisão, onde justificativas nem sempre eram bem explicitadas ou necessárias. Entretanto, a partir do advento da Revolução Industrial no século XVIII, surgiu a divisão do trabalho em níveis hierárquicos, bem como a migração do planejamento e decisões para um nível gerencial. Novas formas de gestão científica foram desenvolvidas e tiveram por consequência a tomada de decisão com base científica, onde devem ser considerados métodos para a tomada de decisão, seus custos e suas consequências para a própria gestão.

O processo de tomada de decisão científico varia de acordo com as especificidades do tema abordado, as informações disponíveis, as possíveis de serem utilizadas, a qualidade delas e do viés da formação profissional do indivíduo tomador de decisão. Dessa forma, este processo é representado em alto nível, com o objetivo de minimizar a perda de informações na sua representação e por conseguinte, utilizar ao máximo a informação disponível. As formas de modelar esse processo variam de acordo com as fontes, suas possíveis representações estatísticas, matemáticas ou computacionais e do contexto sobre o qual ele está inserido. Daí emergem as formas interdisciplinares de modelagem, suas representações e mesmo as suas diversas utilizações em cenários complexos de tomada de decisão.

Particularmente, esse processo é crítico, quando essas decisões são tomadas nas áreas da Saúde, pois envolvem direta ou indiretamente vidas humanas. Desse modo, é importante ao profissional tomador de decisões deter esse conhecimento interdisciplinar para saber reconhecer as implicações da tomada de decisão, avaliá-las e reavaliá-las, sob o prisma da Saúde. É também de particular

1 Departamento de Estatística, Universidade Federal da Paraíba, João Pessoa-PB. e-mail: ronei@de.ufpb.br

2 Programa de Pós-Graduação em Modelos de Decisão e Saúde. Universidade Federal da Paraíba, e-mail: rackynelly@gmail.com 
interesse prever os impactos benéficos ou não dessas decisões sobre o processo, visando aprimorálo continua e ciclicamente privilegiando as boas práticas e mitigando os seus malefícios.

Basicamente, duas grandes áreas são de interesse nesse processo: o primeiro é o desenvolvimento e o processamento de informações em várias áreas da saúde, abrangendo o uso de modelos estatísticos, matemáticos e computacionalmente inteligentes sob uma ótica de suporte à tomada de decisão, desenvolvendo aplicativos que orientem o tomador de decisões nesse processo. $\mathrm{O}$ segundo é a própria aplicação desses modelos em casos e situações próprios da área de Saúde, nas quais há a necessidade de tomada de decisão sobre informações e/ou dados, sejam eles qualitativos ou quantitativos, tanto em formas inovativas no lidar com os pacientes, quanto na própria gestão em Saúde e seus processos particulares.

Isso demonstra a alta relevância da utilização do processo de tomada de decisão científico em Saúde e também o surgimento das várias propostas de ferramentas e técnicas para a sua solução sobre os mais diversos campos da Saúde. Essa Edição Especial é composta por quinze artigos dedicados à aplicações originais, inovadoras e pesquisas recentes sobre modelos de tomada de decisão em Saúde.

O primeiro artigo denominado "Relação entre Síndrome de Burnout e condições de saúde entre militares do exército", co-autorado por Bruno Mendes de Jesus, Sâmela Ribeiro da Silva, Danilo Lima Carreiro, Laura Tatiany Mineiro Coutinho, Christiane Athayde Santos, Andréa Maria Eleutério de Barros Lima Martins e Wagner Luiz Mineiro Coutinho visa a identificação da Síndrome de Burnout entre militares do Exército, a partir de informações sobre as características demográficas, socioeconômicas, de saúde, hábitos de vida/atitudes/comportamentos e fatores ocupacionais.

O Artigo "A vulnerabilidade da infância frente ao excesso de peso: considerações éticas sobre responsabilidades" de Caroline Filla Rosaneli e Thiago Rocha da Cunha discutem, a partir de uma problematização bioética, o problema da obesidade infantil.

O artigo "Bases metodológicas da formação em Fisioterapia: discutindo o distanciamento entre os processos de formação e a utilização da força de trabalho" co-autorado por Daniela Macêdo Pimentel, Cesar Cavalcanti da Silva e Eufrásio de Andrade Lima Neto visa, a partir do uso de teste de hipóteses, buscou-se identificar os pontos de distanciamento entre a formação e a utilização da força de trabalho do fisioterapeuta na atenção básica à saúde em João Pessoa/PB.

O artigo "Modelo de decisão sobre o uso de preservativos: Uma regressão logística multinomial" de Isis Milane Batista, Ana Eloisa Cruz de Oliveira, Josemberg Moura de Andrade e Kaline Silva Lima propõe o uso de um modelo para auxiliar o processo de tomada de decisão sobre a eficácia no uso do preservativo que está relacionada à confiança, que o indivíduo sente no sucesso do seu uso.

O artigo co-autorado por Jackeline Abílio de Souza, Ana Tereza de Medeiros e Ulisses Tempus, actas de saúde colet, Brasília, 10(2), 07-10, jun, 2016. 
Umbelino dos Anjos, intitulado "Fatores associados à violência conjugal em um grupo de mulheres no município de João Pessoa - Paraíba” investiga os fatores associados à violência conjugal em um grupo de mulheres que se apresentaram na Delegacia Especializada de Atendimento à Mulher do município em um período do ano de 2012.

Jane Kelly Oliveira Friestino, Denisa Mendonça, Pedro Oliveira, Carla M. Oliveira, Rosemeire de Olanda Ferraz e Djalma de Carvalho Moreira Filho fazem uso de técnicas de análise espacial baseada no método Bayesiano empírico para identificar padrões espaciais de incidência de cânceres considerados raros na cidade de Campinas-SP no artigo "Estatística Espacial Aplicada aos Estudos de Incidência de Câncer".

O artigo "Spatial analysis of neglected diseases in Brazil, 2007 to 2009" também utiliza métodos de análise espacial para detecção de municípios prioritários de doenças negligenciadas é co-autorado por Joyce Mendes de Andrade Schramm, Mônica Rodrigues Campos, Isabel Cristina Martins Emmerick, Luiz Villarinho pereira Mendes, Jurema Corrêa Mota e Sérgio Henrique Almeida Silva Junior.

O artigo co-autorado por Lavoisier Morais de Medeiros, Ana Maria Gondim Valença e Ulisses Umbelino dos Anjos, cujo título é "Modelo preditivo para diagnóstico da sepse em unidades de terapia intensiva" versa sobre um modelo de decisão probabilista como suporte ao diagnóstico da sepse, obtendo um coeficiente de concordância Kappa quase perfeito.

Liliane dos Santos Machado, Ana Maria Gondim Valença e Alana M. Morais utilizam máquinas de estados finitos como base para um modelo de inteligência no artigo "Um serious game para educação sobre saúde bucal em bebês", cujo foco é a informação para as mães sobre como se deve proceder a higiene oral dos bebês.

Uma discussão sobre aspectos da modelagem em realidade virtual do comportamento hemodinâmico de um paciente virtual cirúrgico, em momentos distintos da indução anestésica, é o foco do artigo "Modelagem do comportamento hemodinâmico de um paciente virtual cirúrgico baseado em sistema especialista fuzzy" de Paulo Farias Paiva, Liliane dos Santos Machado e Tâmela Costa.

Os autores Marianne Louise Marinho Mendes, Fernanda Rodrigues da Silva, Cristhiane Maria Bazílio de Omena Messia, Paulo Gustavo Serafim de Carvalho, Tarcísio Fulgêncio Alves da Silva trouxeram para esse número a discussão dos hábitos alimentares e o nível de atividade física entre alunos de cursos da área da saúde. Esse estudo Esse artigo "Hábitos Alimentares e Atividade Física de Universitários da Área de Saúde do Município de Petrolina-PE” e verificaram a inadequação de hábitos alimentares, e baixo nível de atividade física.

No trabalho "Avaliação da capacidade de vigilância do Centro de Informações Estratégicas em Vigilância em Saúde do Ministério da Saúde, Brasil” dos autores Melina Érica Santos, José Fernando 
de Souza Verani, Vitor Laerte Pinto Junior, Wildo Navegantes Araújo, a partir da aplicação do modelo lógico e considerando alguns indicadores estratégicos, os processos de trabalho do CIEVS/ MS foram avaliados. A avaliação proposta identificou potencialidades e fragilidades da estratégia.

O artigo "Programa de Pós-graduação em Modelos de Decisão e Saúde: uma experiência exitosa na formação em Saúde no Estado da Paraíba" relata a experiência de um Programa de Pós-graduação interdisciplinar envolvendo as Ciências da Saúde e das Exatas cujo objetivo é a formação de mestres e doutores na área de modelos de tomada de decisão com foco em Saúde. Esse relato é co-autorado por Ronei Marcos de Moraes, Rodrigo Toledo Vianna, Rackynelly Alves Sarmento Soares e César Cavalcanti da Silva.

O artigo co-autorado por Thaíse K. L. Costa, Liliane dos Santos Machado, Ana Maria Gondim Valença, Ronei Marcos Moraes, sob o título "Modelo de avaliação para formação em Saúde baseada em jogos e ambientes virtuais" discute o uso de modelos de decisão para avaliar o desempenho de alunos usuários de uma plataforma de jogos e ambientes virtuais para educação em saúde.

Ulanna Maria Bastos Cavalcante, Silvia Adelaide Linhares de Melo, Sabine Helena Dantas, Hemílio Fernandes Campos Coelho e Caliandra Maria Bezerra Luna Lima são os autores do trabalho cujo título é “Avaliação da Qualidade de Vida de pacientes com enteroparasitoses atendidos num Hospital Escola de João Pessoa-PB utilizando um Modelo de Regressão Logística

Por fim, mas não menos importante, como Editores convidados dessa Edição Especial em "Modelos de Decisão aplicados à Saúde: teoria e prática", gostaríamos de agradecer a todos os autores que prestaram sua contribuição e a todos os revisores pelas suas revisões cuidadosas e construtivas que levaram à melhoria da qualidade dos artigos aqui publicados.

Pensando em dar maior visibilidade aos artigos aqui publicados, esse número, apresentou um desafio adicional: é o primeiro publicado na TEMPUS que traz, a maioria dos artigos, em dois idiomas (inglês e Português), seguindo o movimento natural de internacionalização das revistas científicas brasileiras. Desse modo, a partir desse número a TEMPUS adota esse padrão de publicação bilíngue.

Também gostaríamos de agradecer à Ana Valéria Machado Mendonça, Coordenadora da Equipe Editorial da Revista, por aceitar a publicação dessa Edição Especial e pelas valiosas colaborações durante o processo dessa publicação. 